\section{Optical coherence tomography analysis of evolution of Bruch's membrane features in angioid streaks}

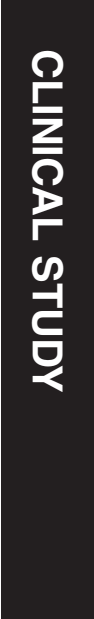

${ }^{1}$ Department of Ophthalmology, IRCCS Ospedale San Raffaele, University Vita-Salute, Milan, Italy

${ }^{2}$ G.B. Bietti Eye Foundation-IRCCS, Rome, Italy

${ }^{3}$ Department of Ophthalmology, University of Magna Graecia, Catanzaro, Italy

Correspondence: G Querques, Department of Ophthalmology, IRCCS Ospedale San Raffaele, University Vita-Salute San Raffaele, Via Olgettina 60, Milan 20132, Italy

Tel: +390226432648 ;

Fax: +390226433643.

E-mail: giuseppe.querques@ hotmail.it

Received: 1 February 2017 Accepted in revised form: 27 April 2017 Published online: 16 June 2017

\begin{abstract}
Purpose To describe optical coherence tomography (OCT) features in the Bruch's membrane (BM) of eyes with angioid streaks (AS) and evaluate their evolution over the follow-up.

Patients and methods Patients with AS presenting between March 2016 and September 2016 at two tertiary referral centers were consecutively recruited in this study. Eligibility criteria included prior spectral domain (SD)-OCT images, taken at least 3 months before at the same referral center, with automated eye tracking and image alignment modules. Alterations of BM were described and compared to previous scans over the follow-up. Multimodal imaging was used to identify alteration of retinal pigment epithelium (RPE) and choroid.

Results Thirty-two eyes of 16 consecutive patients with AS were included. BM undulations, mostly observed around the optic nerve head, were found in $19(59.4 \%)$ of 32 eyes. BM breaks were found in $31(96.9 \%)$ out of 32 eyes. Evolution of BM undulations into BM breaks was observed in 5 eyes (15.6\%). Choroidal neovascularization (CNV) was observed in 12 eyes (37.5\%) during follow-up, typically in areas of BM interruption.

Conclusions BM undulations, probably caused by high stretching forces exerted on the $\mathrm{BM}$ around the optic nerve head, seem to precede some BM breaks. BM interruptions may be a preferred way for the growth of $\mathrm{CNV}$, which was identified in one-third of our cases.
\end{abstract}

Eye (2017) 31, 1600-1605; doi:10.1038/eye.2017.112; published online 16 June 2017

\section{Introduction}

In 1917, Kofler supposed that Bruch membrane (BM) was the anatomic location of angioid
A Marchese ${ }^{1}$, M Parravano², A Rabiolo', A Carnevali ${ }^{1}{ }^{3}$, E Corbelli ${ }^{1}$, MV Cicinelli ${ }^{1}$, M Battaglia Parodi', L Querques ${ }^{1}$, F Bandello ${ }^{1}$ and G Querques ${ }^{1}$ streaks (AS), ${ }^{1}$ basing his observation on clinical findings only. Histological studies then revealed ruptures of BM exactly at the AS site. ${ }^{2,3}$ Recently, structural optical coherence tomography (OCT) confirmed the presence of these BM interruptions in vivo, reporting also a variety of other OCT features consistently associated with AS, including (i) hyper-reflective dots in the BM, (ii) $\mathrm{BM}$ undulations, and (iii) large $\mathrm{BM}$ dehiscences. ${ }^{4,5}$ Hyper-reflective dots in BM were observed on spectral domain OCT scans and have been correlated to increased mineralization of BM. ${ }^{4}$ This alteration reduces the elasticity of this basal membrane and its ability to absorb stretching forces. Therefore, deformation of BM, named BM undulations, are often associated with AS on structural OCT images. ${ }^{6}$

AS have a complex pathogenesis being related to many clinical conditions. Among patients with AS, $50-80 \%$ are affected by Pseudoxanthoma Elasticum (PXE), an inherited disease due to the mutation of ABCC6 gene. ${ }^{7}$ This gene encodes for a membrane transporter protein, mainly expressed in liver and kidneys. Through ABCC6, factors able to inhibit the calcification of elastic fibers are secreted into the blood. ${ }^{8}$ Other conditions associated with AS, which may interfere with calcium homeostasis, are Paget disease of bone and sickle cell anemia. Both conditions may promote mineralization of extracellular matrix, including BM. ${ }^{9,10}$ These metabolic alterations affecting BM have been extensively described, however, their evolution during time has not been documented yet.

The aim of this study was to describe the structural OCT features of BM in patients with AS and evaluate if these findings may evolve from pre-existing BM abnormalities over the follow-up. 


\section{Patients and methods}

Patients affected by AS presenting between March 2016 and September 2016 at two high-volume referral centers (the Medical Retina \& Imaging Unit of the Department of Ophthalmology, University Vita-Salute San Raffaele in Milan, Italy, and the G.B. Bietti Eye Foundation-IRCCS, Rome, Italy) were consecutively recruited in this observational retrospective longitudinal study. The study was conducted in agreement with the Declaration of Helsinki for research involving human subjects. All patients signed a written general consent to participate to observational studies, which was approved by the ethics committee of San Raffaele Hospital.

Inclusion criteria for the study were diagnosis of AS; age: $>18$ years old; sufficiently clear ocular media, adequate pupillary dilation, and fixation to permit highquality OCT imaging; prior spectral domain (SD)-OCT images, taken at least 3 months before at the same referral center, with automated eye tracking and image alignment modules.

Exclusion criteria consisted of any eye disease other than AS, including age-related macular degeneration (AMD), retinal vascular diseases, vitreoretinal diseases, history of central serous retinopathy or retinal detachment; previous ocular surgeries except from uneventful cataract extraction $>6$ months prior to OCT images acquisition.
Each patient underwent a comprehensive ophthalmologic examination, including measurement of best-corrected visual acuity (BCVA), anterior segment biomicroscopic, and dilated fundus examination, SD-OCT (Spectralis+HRA; Heidelberg Engineering, Heidelberg, Germany). SD-OCT protocol included 19 horizontal raster, 49 horizontal raster dense linear, and six radial linear B-scans, centered on the fovea and on the optic disc.

A masked examiner (AM) retrospectively compared the OCT features to those available from previous scans over the follow-up. BM undulation was defined as outward and/or inward deformations at the level of the BM (Figure 1). BM breaks were defined as interruptions of BM (Figures 1 and 2). Large dehiscences of BM were defined as any defect at the BM greater than $250 \mu \mathrm{m}$, not contiguous to any visible streaks (Figure 3). ${ }^{4}$ Hyperreflective material, suggesting fibrosis or fibrovascular membranes, was defined as abnormal tissue over, through or beneath the RPE-Bruch's membrane complex (Figure 4). The presence of CNV was assessed on the basis of multimodal imaging with fluorescein angiography (FA), indocyanine angiography (ICGA), blue-light autofluorescence (BAF), and OCT-angiography (AngioPlex CIRRUS HD-OCT model 5000, Carl Zeiss Meditec, Inc., Dublin, OH, USA). However, this multimodal imaging was not available for each follow-up visit, where only SD-OCT and charts were reviewed.
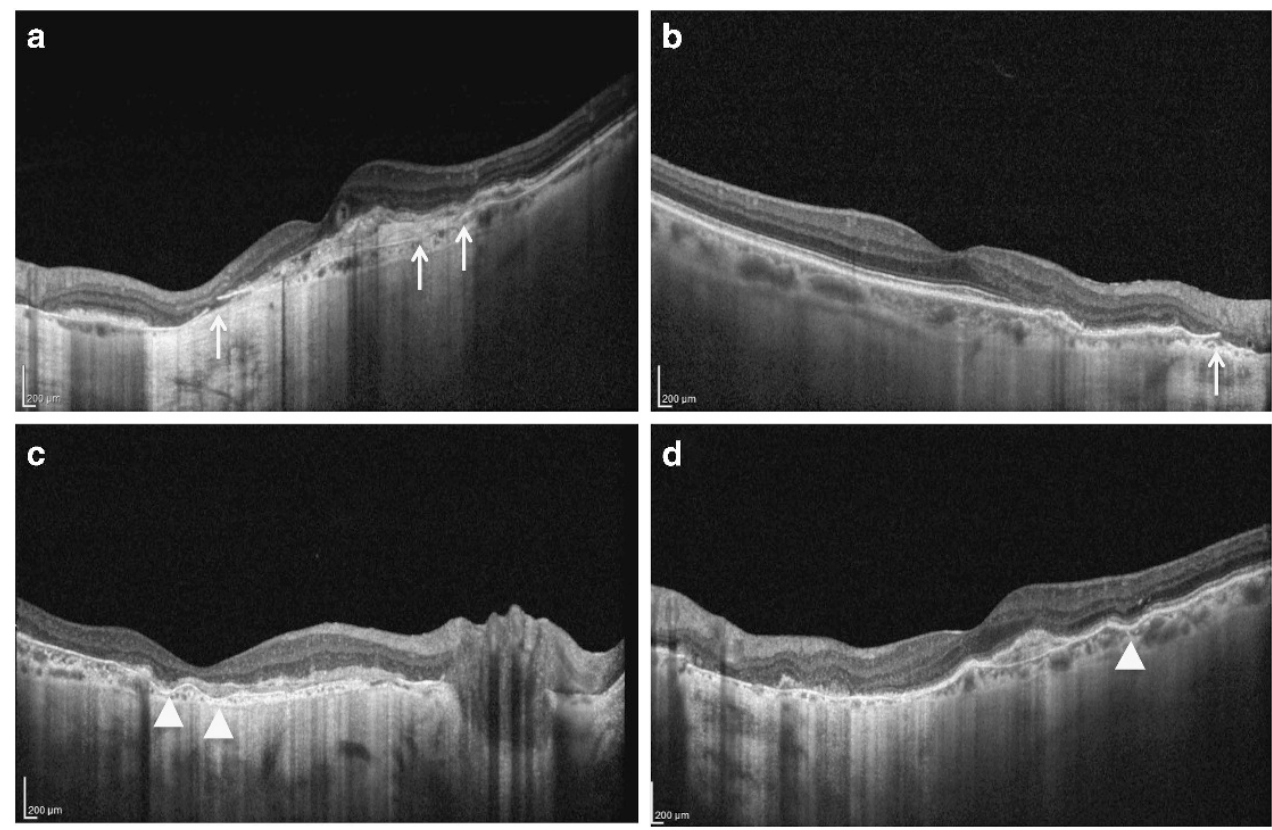

Figure 1 Spectral domain optical coherence tomography (SD-OCT) of Bruch's membrane (BM) breaks and BM undulations in patients with angioid streaks (AS). Bruch's breaks, seen as interruptions of BM (arrows), were easier to identify in case of atrophy of retinal pigment epithelium (a), left arrow and panel (b). Bruch's undulations (arrowheads) (c, d) were identified mainly under hyper-reflective material or close to the optic disc. 


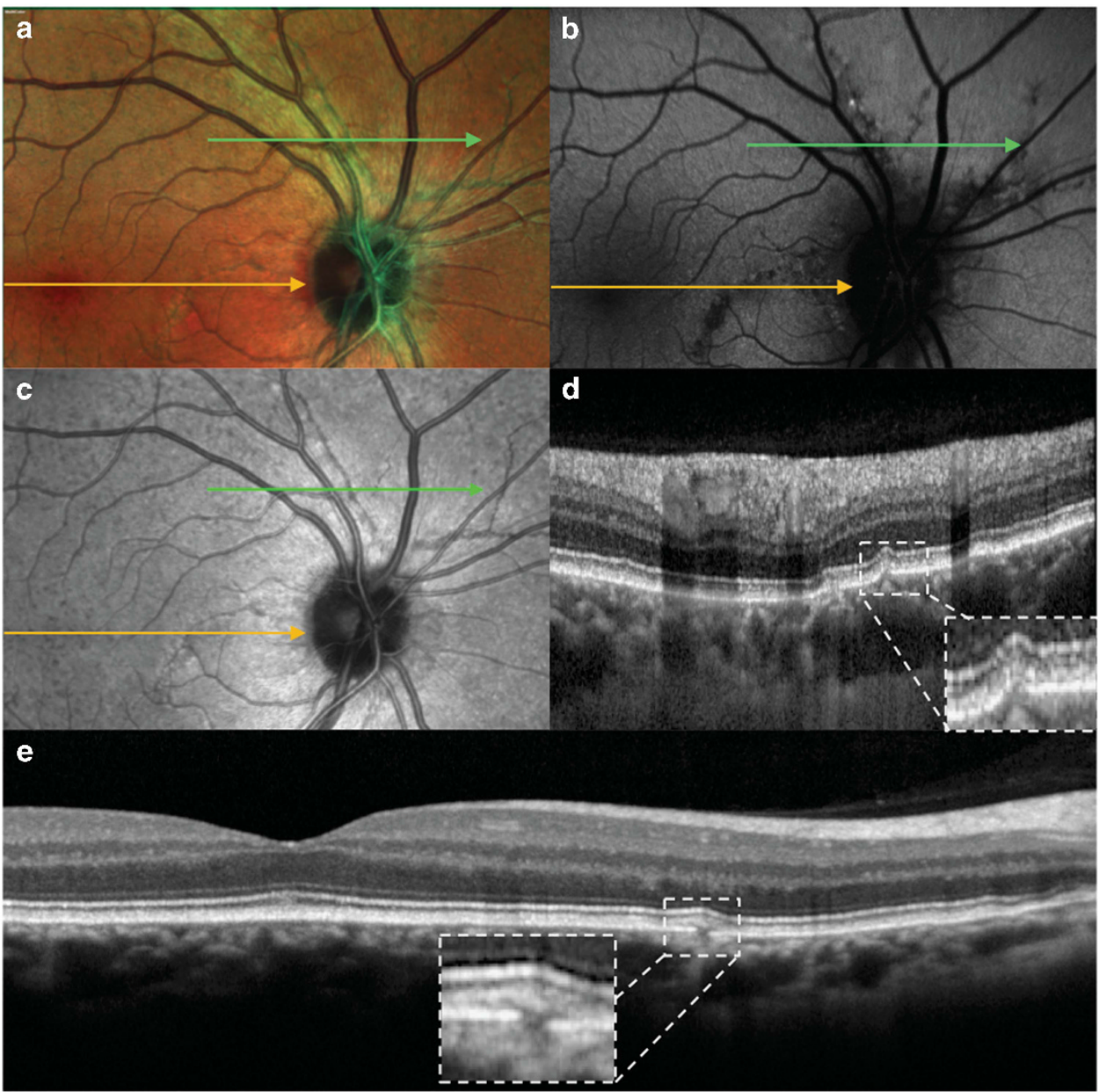

Figure 2 Multimodal imaging of Bruch's membrane (BM) breaks in a patient with angioid streaks (AS). Multicolor (a), blue autofluorescence (b), and infrared fundus (c) images of AS corresponding to small BM breaks on OCT (d and e, enlarged sections). Superimposed green arrows correspond to the OCT scan of panel (d), while orange arrows to the OCT scan of panel (e).

Descriptive statistical analyses were performed using SPSS version 22.0 (SPSS, Inc., Chicago, IL, USA). Variable included in the analysis were: age, sex, race, number and type of BM lesions detected by means of SD-OCT. All the data were expressed as mean $\pm \mathrm{SD}$.

\section{Results}

Thirty-two eyes of 16 patients (8 female and 8 males; mean age $65.8 \pm 13.9$ years) were included in this study. The demographic data are presented in Table 1. Twelve patients $(75 \%)$ were diagnosed with PXE on the basis of skin biopsy results and/or genetic analysis, 1 (6.3\%) with sickle cell disease by means of hemoglobin electrophoresis and $3(18.8 \%)$ with idiopathic AS.

The mean follow-up time for SD-OCT references was 23.5 months $( \pm 13.2)$. The mean number of SD-OCT examinations per patient was $4.1( \pm 3.4)$.
Bruch's undulations were identified in 19 out of 32 eyes $(59.4 \%)$, typically under fibrovascular material or close to the optic disc (Figure 1).

Bruch's breaks were noted in 31 (96.9\%) out of 32 eyes on SD-OCT (Figures 1 and 2). In older patients, with alteration of the overlying retinal pigment epithelium (RPE) and outer retinal atrophy, it was easier to identify the interruption in the BM compared to younger patients with an intact RPE.

Large dehiscences ( $>250 \mu \mathrm{m}$ ) in BM, not contiguous with AS, were observed in 9 (28.1\%) out of 32 eyes: five were in the context of peripapillary atrophy, and four under actively leaking CNV or hyper-reflective material, suggesting proliferating fibrovascular membranes (Figure 3) evolving toward subretinal fibrosis.

In 5 eyes (15.6\%), we observed a progression of BM undulations into BM breaks using previous OCT scans of the same section as references (Figure 3). All BM breaks developed in areas under high mechanical stress of 


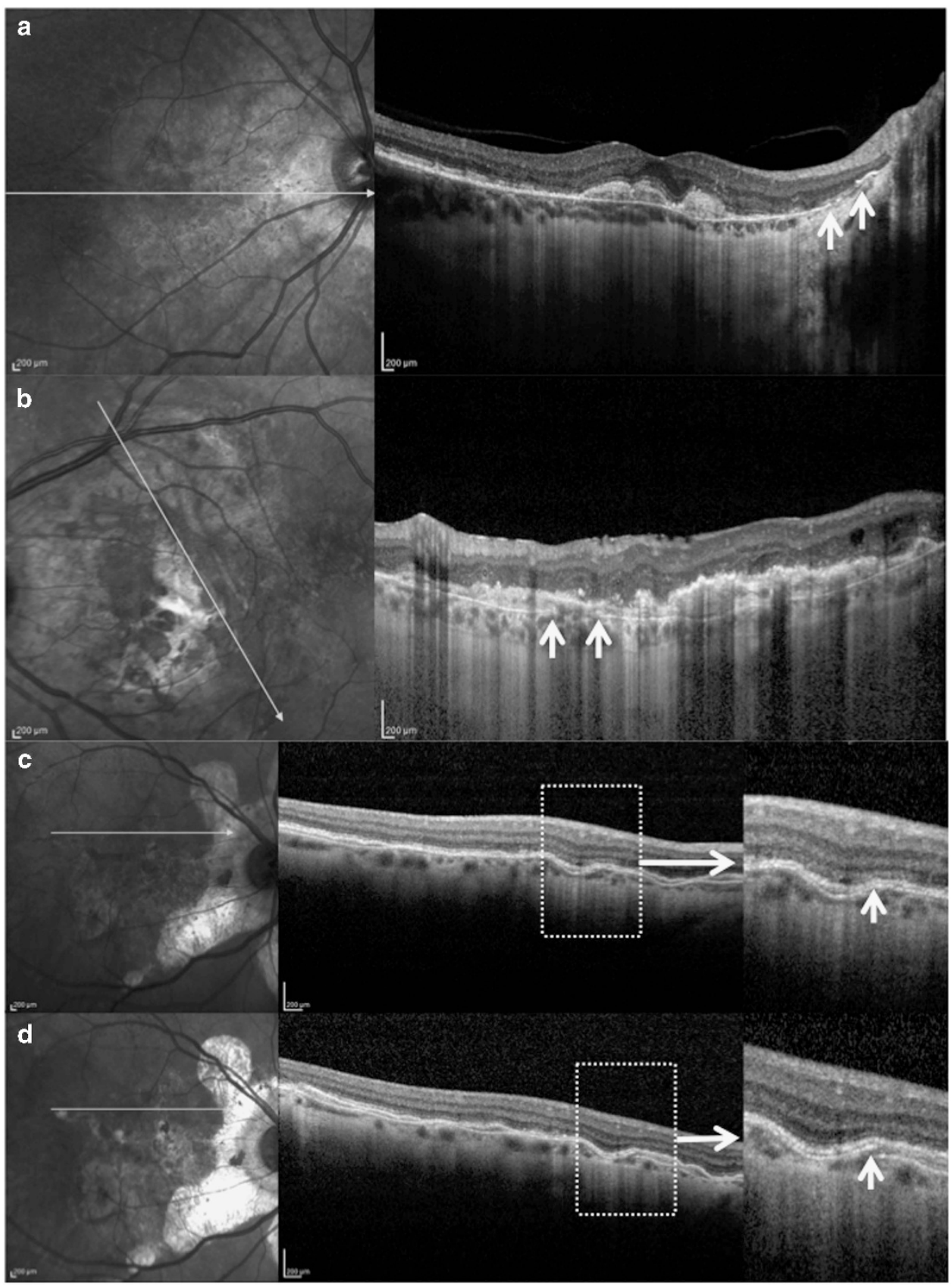

Figure 3 Spectral domain optical coherence tomography (SD-OCT) of large dehiscences ( $>250 \mu \mathrm{m})$ in Bruch's membrane (BM) and of undulations evolving into BM breaks in patients with angioid streaks (AS). Large dehiscences ( $>250 \mu \mathrm{m})$ in BM, not contiguous with AS, were observed in area of parapapillary atrophy (a) or under active or quiescent CNV or fibrovascular material (b). Using SD-OCT previous sets of references, a progression of BM undulations (c) into BM breaks (d) was observed.

undulated $\mathrm{BM}$ (that is, vertex and margins), after a mean follow-up of 8.4 months ( \pm 6.2 ). Hyper-reflective material, indicating fibrovascular membranes was typically present above and through breaks of BM (Figure 4).

Through multimodal imaging techniques (FA, ICGA and OCTA), choroidal neovascularization (CNV) were detected in 12 eyes (37.5\%) during follow-up, 10 in the macular area and 2 in the peripapillary area (within 1 disc diameter from the optic head). BM undulations were present in 8 out of 12 eyes with CNV (66.7\%), and 11 out of 20 eyes without CNV (55\%).

\section{Discussion}

AS are breaks in the BM with or without involvement of RPE or choriocapillaryis. In accordance to an electron 


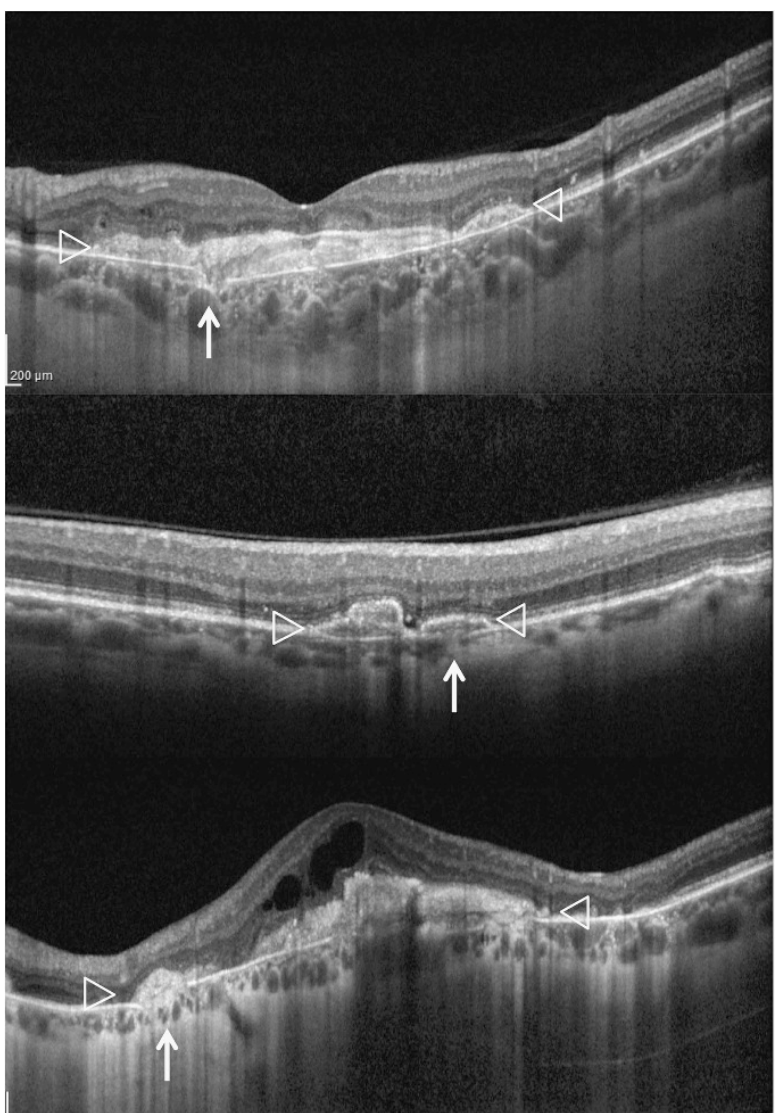

Figure 4 Spectral domain optical coherence tomography (SDOCT) images of hyper-reflective material above and through interruptions of Bruch's membrane (BM) in patients with angioid streaks (AS). Hyper-reflective material (inside arrowheads) was often present above and through interruptions of BM (arrows) or under active or quiescent CNV (middle panel) or fibrovascular material (upper and lower panels).

Table 1 Main demographics and types of Bruch's membrane lesionsin patients with angioid streaks

\begin{tabular}{lc}
\hline Sex, $\mathrm{n}$ & \\
$\quad$ Male & 8 \\
Female & 8 \\
& \\
Age, $\mathrm{y}$, mean $\pm \mathrm{SD}$ & $65.8 \pm 13.9$ \\
Total eyes & 32 \\
BM lesions visible with SD-OCT, $\mathrm{n}$ of eyes $(\%)$ & \\
BM undulations & $19(59.4 \%)$ \\
BM breaks & $31(96.9 \%)$ \\
Large dehiscences of BM $(>250 \mu \mathrm{m})$ & $9(28.1 \%)$ \\
Choroidal neovascularization & $12(37.5 \%)$ \\
Evolution of BM undulation into BM breaks & $5(15.6 \%)$ \\
\hline
\end{tabular}

Abbreviations: BM, Bruch's membrane; SD-OCT, spectral domain optical coherence tomography; $y$, years.

microscopy study, BM breaks initially do not cause alterations of RPE and choriocapillary layers. ${ }^{6}$ Large and evoluted breaks are accompanied by RPE atrophy, choriocapillary thinning, and ingrowth of fibrous tissue. ${ }^{11}$
The first step in AS formation is the conversion of BM from an elastic structure to a brittle one. Thickening and increased basophilia reflecting abnormal calcification of the BM has been demonstrated ex vivo though histological studies. $^{2}$ These observations have been confirmed in vivo using OCT as hyper-reflectivity and thickening of BM/ RPE complex. ${ }^{4} \mathrm{BM}$ calcification occurs prior to AS onset and, conversely, AS develop in calcified areas. It has been hypothesized that brittle BM is more vulnerable to mechanical forces due to its reduced elasticity and, thus, it is prone to develop ruptures even with physiological tensions (for example, extraocular muscles contraction, minor trauma, intraocular pressure spike). In accordance to this mechanical theory, AS usually occur in loci minoris resistentiae, as the peripapillary area, due to several factors, including that (i) the optic nerve head is the hub for the movements of the globe; (ii) BM is physiologically interrupted in the peripapillary area; (iii) retinal vessels spreads from the optic nerve cup to the peripheral retina pushing on outer retinal layers, and sometimes, on BM itself.

$\mathrm{BM}$ undulations are a common finding in patients affected by AS (Figure 1). BM undulations have been firstly observed in histopathologic studies, even though it was unclear whether they were real findings or artifacts (for example, postmortem changes, histological fixation), and subsequently confirmed by OCT studies. Although undulations have been reported also in patients affected by neovascular AMD, they are six times more frequent and more prominent in patients with $\mathrm{CNV}$ secondary to AS. ${ }^{6}$

Interestingly, we observed the progression from BM undulations to breaks (Figure 3). We hypothesize that BM undulations represent points of reduced resistance at higher risk of rupture over the follow-up. Indeed, BM breaks developed at the undulation vertex and margins, probably points subjected to high mechanical stress.

In a study by Ellabban et al, ${ }^{6} \mathrm{BM}$ undulations were found in about 73 and $38 \%$ of AS eyes with or without $\mathrm{CNV}$, respectively. In our study, we also found an increased prevalence of BM undulations in eyes with $\mathrm{CNV}$ compare to those without $\mathrm{CNV}$, although the difference between these two groups was less pronounced.

Large dehiscences in BM were described by Spaide and Jonas $^{5}$ in peripapillary atrophic areas in eyes affected by PXE. In our study, we observed large dehiscences in BM not only in atrophic areas around the optic head but also in the macular area (Figure 3). We hypothesize that all these BM interruptions may be a preferred way for the growth of $\mathrm{CNV}$, which was identified in one-third of our cases. The development of fibrovascular membranes in correspondence of BM breaks and large dehiscences could 
be also interpreted as a tentative reparative process leading to the development of neovessels.

We are aware that the present study has several limitations, including the number of patients, the coexistence of many underlying diseases associated with AS, and the retrospective design. Furthermore, the evolution of undulations into breaks of Bruch's membrane was documented only on the basis of OCT images; these could suffer from several artifacts, as in the evaluation of BM interruptions under thick hyperreflective material (for example, $\mathrm{CNV}$ ). Despite these limitations, our study reveals that many subforms of BM interruption exist, potentially, leading to the development of CNV and atrophic changes.

In conclusion, mechanical stress exerted on a brittle BM seems to be a major putative factor in AS development. In this study, we reported how BM undulations may evolve into BM ruptures and, thus, they represent loci minoris resistentiae of the $\mathrm{BM}$ itself. In addition, we describe large $\mathrm{BM}$ dehiscences not contiguous to AS within the macular region.

Further studies are warranted in order to explore the evolution of BM breaks and to investigate their association with $\mathrm{CNV}$.

\section{Summary}

What was known before

- Angioid streaks may be associated with ruptures, undulation and large dehiscences of Bruch's membrane.

- Choroidal neovascularization (CNV) and atrophy may complicate angioid streaks.

What this study adds

- Mechanical stress exerted on weaker points (e.g. undulations) of a brittle Bruch's membrane seems to be a major putative factor in angioid streaks development.

- Undulations of Bruch's membrane may evolve into ruptures, potentially leading to the development of $\mathrm{CNV}$ and atrophic changes.

\section{Conflict of interest}

The authors declare no conflict of interest.

\section{Acknowledgements}

MP has the following financial disclosures: Allergan (C), Bayer (S); Novartis (S). MBP has the following disclosures:
Bausch and Lomb (C). GQ has the following disclosures: Allergan (S), Bayer (S); Novartis (S), Zeiss (S), Allergan $(\mathrm{C})$, Alimera (C), Bausch and Lomb (C), Novartis (C), Bayer (C), Heidelberg (C), Zeiss (C). FB has the following disclosures: Allergan (S), Alimera (S), Bayer (S), FarmilaThea (S), Schering Pharma (S), Sanofi-Aventis (S), Novagali (S), Pharma (S), Hoffmann-la Roche (S), Genetech (S), Novartis (S).

\section{References}

1 Koefler A. Beitrage zur Kenntnis der angioid Streaks. Arch Augenheilkd 1917; (82): 134-149.

2 Dreyer R, Green WR. The pathology of angioid streaks: a study of twenty-one cases. Trans Pa Acad Ophthalmol Otolaryngol 1978; 31(2): 158-167.

3 Jensen OA. Bruch's membrane in pseudoxanthoma elasticum. Histochemical, ultrastructural, and x-ray microanalytical study of the membrane and angioid streak areas. Albrecht Von Graefes Arch Klin Exp Ophthalmol 1977; 203(3-4): 311-320.

4 Charbel Issa P, Finger RP, Holz FG, Scholl HP. Multimodal imaging including spectral domain OCT and confocal near infrared reflectance for characterization of outer retinal pathology in pseudoxanthoma elasticum. Invest Ophthalmol Vis Sci 2009; 50(12): 5913-5918.

5 Spaide RF, Jonas JB. Peripapillary atrophy with large dehiscences in bruch membrane in pseudoxanthoma elasticum. Retina 2015; 35(8): 1507-1510.

6 Ellabban AA, Hangai M, Yamashiro K, Nakagawa S, Tsujikawa A, Yoshimura N. Tomographic fundus features in pseudoxanthoma elasticum: comparison with neovascular age-related macular degeneration in Japanese patients. Eye (Lond) 2012; 26(8): 1086-1094.

7 Bergen AA, Plomp AS, Schuurman EJ, Terry S, Breuning M, Dauwerse $\mathrm{H}$ et al. Mutations in ABCC6 cause pseudoxanthoma elasticum. Nat Genet 2000; 25(2): 228-231.

8 Jiang Q, Oldenburg R, Otsuru S, Grand-Pierre AE, Horwitz EM, Uitto J. Parabiotic heterogenetic pairing of Abcc6-/-/ Rag1-/- mice and their wild-type counterparts halts ectopic mineralization in a murine model of pseudoxanthoma elasticum. Am J Pathol 2010; 176(4): 1855-1862.

9 Jampol LM, Acheson R, Eagle Jr RC, Serjeant G, O'Grady R. Calcification of Bruch's membrane in angioid streaks with homozygous sickle cell disease. Arch Ophthalmol 1987; 105(1): 93-98.

10 Kerr NM, Cassinelli HR, DiMeglio LA, Tau C, Tuysuz B, Cundy $\mathrm{T}$ et al. Ocular manifestations of juvenile Paget disease. Arch Ophthalmol 2010; 128(6): 698-703.

11 Gliem M, Zaeytijd JD, Finger RP, Holz FG, Leroy BP, Charbel Issa P. An update on the ocular phenotype in patients with pseudoxanthoma elasticum. Front Genet 2013; 4: 14 . 